Revue d'histoire de l'Amérique française

REVUE D.HISTOIRE DE L'AMÉRIQUE FRANÇAISE

\title{
PENDERGAST, James F. et Bruce G. TRIGGER, Cartier's Hochelaga and the Dawson Site. McGill-Queen's University Press, Montreal-London, 1972. xxvii-388 p. \$25.00.
}

\section{Lucien Campeau}

Volume 27, numéro 1, juin 1973

URI : https://id.erudit.org/iderudit/303251ar

DOI : https://doi.org/10.7202/303251ar

Aller au sommaire du numéro

Éditeur(s)

Institut d'histoire de l'Amérique française

ISSN

0035-2357 (imprimé)

1492-1383 (numérique)

Découvrir la revue

Citer ce compte rendu

Campeau, L. (1973). Compte rendu de [PENDERGAST, James F. et Bruce G. TRIGGER, Cartier's Hochelaga and the Dawson Site. McGill-Queen's University Press, Montreal-London, 1972. xxvii-388 p. \$25.00.] Revue d'histoire de l'Amérique française, 27(1), 112-113. https://doi.org/10.7202/303251ar d'utilisation que vous pouvez consulter en ligne.

https://apropos.erudit.org/fr/usagers/politique-dutilisation/ 
Pendergast, James F. et Bruce G. Trigger, Cartier's Hochelaga and the Dawson Site. McGill-Queen's University Press, Montreal-London, 1972. XXVII-388 p. $\$ 25.00$

Ce livre est dû à la collaboration d'un anthropologue, M. Trigger, et d'un archéologue, M. Pendergast. Des savants réputés y ont contribué: M. W. N. Fenton, qui a écrit l'avant-propos, et MM. MacNeish, Kidd et Anderson, qui ont fourni des notes et des remarques. Le but de l'ouvrage est de réévaluer scientifiquement, à partir des notes de sir John William Dawson et des éléments recueillis par lui lors des fouilles qu'il a faites sur un site préhistorique en 1860, la signification de cette découverte. Ce site, appelé le site Dawson, se trouvait au sud de la rue Sherbrooke, entre les rues Mansfield et Metcalfe. Dawson y trouva des ossements humains, des restes de nourriture, des fragments de poterie, des morceaux de pipes en argile cuite et divers instruments faits d'os ou de pierre, outre quelques rares pièces de métal à destination énigmatique. Dawson avait identifié ce site avec celui du bourg d'Hochelaga, visité par Jacques Cartier, le 3 octobre 1535 .

Le livre est en six parties. $1^{\circ}$ Hochelaga: History and Ethnohistory, par B. G. Trigger; $2^{\circ}$ An Analysis of the Dawson Site Archaeological Material, par J. F. Pendergast; $3^{\circ}$ Archaeological Comparison of Dawson Site with other Iroquoian sites in Eastern Ontario, par le même; $4^{\circ}$ Comments on the Archaeology of the Dawson Site, par R. S. MacNeish; 5० Dawson Site Physical Anthropology, par J. E. Anderson; $6^{\circ}$ Contact Material from the Dawson Site, par K. E. Kidd. Suivent cinq appendices: textes de Jacques Cartier, J. W. Dawson et W. D. Lighthall. Donc un riche matériel, traité avec un grand souci de précision et de méthode.

La première conclusion à retenir semble être que l'identification avec Hochelaga a été trop hâtive, qu'elle n'est pas prouvée et qu'elle est même invraisemblable: il y a eu plusieurs agglomérations de populations indigènes sur l'île de Montréal au seizième siècle. Mais il est confirmé que le site Dawson a été habité par une population de culture iroquoienne, ce que 
déjà la documentation historique indiquait. La présence de pièces de métal, si rares qu'elles soient, indiquerait aussi la contemporanéité de ce site avec une fréquentation française du continent, c'est-à-dire le seizième siècle, s'il était assuré qu'elles appartiennent vraiment au même niveau archéologique que le reste. L'épreuve au carbone-14 indique aussi en gros la même époque. La forme des aiguilles, faites d'os, suggérerait également une influence européenne. Ainsi, le site Dawson serait le plus récent établissement iroquoien préhistorique retrouvé et précéderait de peu l'abandon du Saint-Laurent par les peuples de cette culture, au cours de la seconde moitié du même siècle.

Nous savons gré aux auteurs d'avoir réexaminé tout ce matériel. Les illustrations, les graphiques et les tableaux abondants seront sans doute très utiles aux connaisseurs, non moins que l'intéressante analyse des artefacts, faite par M. Pendergast. Pour ma part, je contesterais plus d'un point de la discussion ethno-historique de M. Trigger.

Ce dernier opte résolument pour la thèse de $\mathrm{M}$. Lanctot relative au débarquement de Jacques Cartier au sud de l'île de Montréal. Celle de M. Beaugrand-Champagne est écartée. J'ai relu les récits de Cartier sur ce sujet et je suis plus que jamais convaincu que ces textes sont inintelligibles dans l'optique de Lanctot, alors qu'ils redeviennent cohérents dans celle de Beaugrand-Champagne, qui fait débarquer le découvreur au pied du Sault-au-Récollet. Mais ce n'est pas le lieu de reprendre la controverse.

M. Trigger s'efforce d'expliquer l'évacuation des tribus iroquoiennes hors du bassin du Saint-Laurent. Il rejette toutes les explications fondées sur les traditions indigènes du $17^{\mathrm{e}}$ siècle, à la vérité incohérentes et discordantes, mais toutes d'accord pour attribuer ces bouleversements à des facteurs internes au monde indigène.

M. Trigger substitue à ces explications traditionnelles une théorie toute fondée sur l'apparition du commerce européen, dont les tribus, à l'envi l'une de l'autre, se seraient disputé les avantages. C'est plausible, cohérent, possible. Mais c'est une spéculation sans appui historique suffisant. Un commerce français considérable et régulier à Tadoussac ne paraît pas antérieur à 1575 , ce qui est probablement trop tard pour donner un fondement à l'hypothèse. Il n'est pas prouvé que Stadaconé et Hochelaga aient été hostiles jusqu'à la guerre, que les Montagnais aient été en situation dominante du fait du commerce et de l'armement européen, que les Hochelagans, dépourvus d'armes importées, aient été en mesure de détruire Stadaconé, que les Iroquois aient eu des motifs commerciaux d'envahir le Saint-Laurent au début. Mieux encore, les découvertes archéologiques faites jusqu'ici ne laissent pas croire que les importations européennes vers l'intérieur aient atteint un volume capable de justifier elles seules ces bouleversements.

Le mérite de l'ouvrage, à mon avis, réside dans la présentation et l'analyse des faits archéologiques et anthropologiques. En ce domaine, il reste encore beaucoup trop à faire pour qu'on puisse dresser avec quelque chance de succès une ethno-histoire de la vallée du Saint-Laurent. 